\title{
Predicting deterioration in patients with lobar haemorrhages
}

\author{
Kelly D Flemming, Eelco F M Wijdicks, Erik K St Louis, Hongzhi Li
}

\begin{abstract}
Objective-To study the clinical course and determine predictors of deterioration in patients with lobar haemorrhages). Methods-A comprehensive review of 61 consecutive patients with lobar haemorrhages was performed. Neurological deterioration was defined as (1) decrease in Glasgow coma sum score by 2 points, (2) new neurological deficit, or (3) clinical signs of brain herniation. A univariate logistic regression was performed and expressed in odds ratios.
\end{abstract}

Results-Sixteen of $61(26 \%)$ patients with lobar haemorrhages deteriorated after admission. In a univariate analysis, only a Glasgow coma score $<14$ predicted deterioration $(75 \%$ of deteriorators $v 24 \%$ who did not deteriorate; $\mathbf{p}<\mathbf{0 . 0 0 0 1}$ ). Initial CT characteristics predictive of deterioration included haemorrhage volume $>60 \mathrm{ml}(63 \% v$ $16 \%, p<0.0001)$, shift of the septum pellucidum $(75 \% v 36 \%, p<0.01)$, effacement of the contralateral ambient cistern $(33 \% v 0 \%, p<0.0001)$, and widening of the contralateral temporal horn $(50 \% v 0 \%$, $\mathbf{p}<0.0001)$. Patients presenting and deteriorating within 12 hours of ictus declined due to enlargement of the haemorrhage. Those who deteriorated more than 12 hours after initial neurological symptoms, showed increased mass effect secondary to oedema.

Conclusion-Patients with lobar haemorrhages presenting immediately after ictus are at risk for deterioration from enlargement of the haemorrhage and predictors of deterioration may be absent. Patients with large volume lobar haemorrhages presenting to the emergency department with decreased level of consciousness and shift on CT are at risk for further deterioration from worsening oedema. These patients require close observation and early aggressive management may be warranted.

(F Neurol Neurosurg Psychiatry 1999;66:600-605)

Keywords: coma; intracerebral haemorrhage; CT; outcome; emergency room

Patients who deteriorate after intracerebral haemorrhage have worse outcomes and increased mortality than those who remain stable after admission. ${ }^{1-8}$ Early prediction of neurological deterioration after intracerebral haemorrhage is important in triage decisions made at initial presentation. Most studies have preferentially analysed patients with ganglionic haemorrhages. ${ }^{12}$ No study has analysed pre- dictors of deterioration in patients with supratentorial lobar haemorrhages, a unique group which are more surgically accessible than deeper haemorrhages.

It has been speculated that early deterioration in patients with intracerebral haemorrhage is secondary to enlargement of the haemorrhage or development of hydrocephalus, whereas late deterioration is linked to perilesional oedema. ${ }^{1}$ We sought to elucidate the frequency, timing, clinical and CT predictors and probable mechanism of deterioration in patients with lobar haemorrhage by analysing admission clinical and CT profiles.

\section{Methods}

PATIENT SELECTION

A total of 3432 patients with the diagnostic code of intracerebral haemorrhage from the Mayo Clinic patient database between 1973 and 1996 were retrospectively reviewed. Of these, 1145 were identified as having lobar haemorrhages. Selection criteria included: (1) admission within 48 hours of the initial neurological deficit; (2) lobar haemorrhage documented by CT; (3) Glasgow coma scale (GCS) sum score $\geqslant 8$. We excluded patients with angiographic evidence of an aneurysm or CT with features suggestive of an aneurysmal rupture, haemorrhagic infarction, haemorrhage into a brain tumour or metastasis, concurrent history of trauma, or those without an initial CT. Because the clinical course may be different in patients with a known coagulopathy, or use of anticoagulant or thrombolytic drug, these patients were also excluded. Sixty one patients (33 male; 28 female) were included in our series. These patients were then divided into those who remained stable and those who deteriorated neurologically. Neurological deterioration was defined as (1) decrease in the GCS sum score by 2 points, or (2) new deficit, or (3) signs of herniation (unilateral mydriasis $>5 \mathrm{~mm}$ or unilateral decorticate or decerebrate rigidity).

\section{CLINICAL DATA}

The following historical features were analysed: age, sex, history of diabetes mellitus, hypertension, treatment for hypertension, cardiovascular disease (myocardial infarction, coronary artery disease, valvar heart disease, arrhythmia, congestive heart failure), dementia, drug use, alcohol misuse, tobacco use, prior intracerebral haemorrhage or ischaemic stroke, use of antiplatelet agent at time of haemorrhage, and family history of intracerebral haemorrhage. The approximate time from the ictus to presentation in the emergency department was recorded. Clinical features at onset 
were recorded including headache, vomiting, seizures, focal deficit, and reduced level of consciousness. Admission examination data as recorded by a neurologist or neurosurgeon were abstracted from emergency room records. Data included: systolic and diastolic blood pressure, pulse, tachypnoea (respiratory rate $\geqslant 30$ breaths per minute), and initial GCS. Abnormality in pupil size and reactivity, corneal reflexes, eye movements, visual field, language, motor examination (defined as facial or limb weakness), sensory examination (defined as hemisensory loss, hemineglect, or astereognosis), and presence of herniation signs.

CT DATA

The side and location (defined by the lobe in which the maximum diameter of the haemorrhage was present) of the haemorrhage were determined. The volume of the haemorrhage was calculated using the volume for an ellipse $(4 / 3 \pi(\mathrm{A} / 2)(\mathrm{B} / 2)(\mathrm{C} / 2))$ as described by Kothari et $a l .{ }^{9}$ The density (homogenous or nonhomogenous), relative size of the ipsilateral and contralateral perimesencephalic cisterns, and horizontal displacement of the pineal gland and septum pellucidum were recorded. Presence of intraventricular blood and ventricular effacement was recorded. The CT was reviewed (KDF; EFMW) without knowledge of the clinical findings.

NEUROLOGICAL DETERIORATION

If a patient deteriorated, the approximate time after ictus was recorded. Repeat neurological examination, CT (if available), and laboratory studies were abstracted.

LABORATORY, DIAGNOSTIC PROCEDURES, THERAPEUTICS

Complete blood count, electrolytes, urea, creatinine, liver enzymes, prothrombin time, partial thromboplastin time, and sedimentation rate were obtained from laboratory summary records. Diagnostic procedures and therapies performed were recorded and included: MRI, angiogram, endotracheal intubation, hyperventilation, steroids, antiepileptic medications, osmotic diuretics, ventricular drainage, and craniotomy.

OUTCOME

Systemic complications (cardiac, pulmonary, or infectious) were abstracted from the hospital course. A modified GCS was used and applied to patients at discharge or death. ${ }^{10}$ The following scale was used: $1=$ death; $2=$ vegetative state (unable to interact with the environment), $3=$ severe disability (unable to live independently, but able to follow commands), $4=$ moderate disability (capable of living independently, but unable to return to work), $5=$ mild or no disability (able to return to work or previous lifestyle).

STATISTICAL ANALYSIS

Data were analysed using the $\chi^{2}$ test or Fisher's exact test. Odds ratios together with $95 \%$ confidence intervals (95\%CIs) were obtained using univariate logistic regression or the exact method.

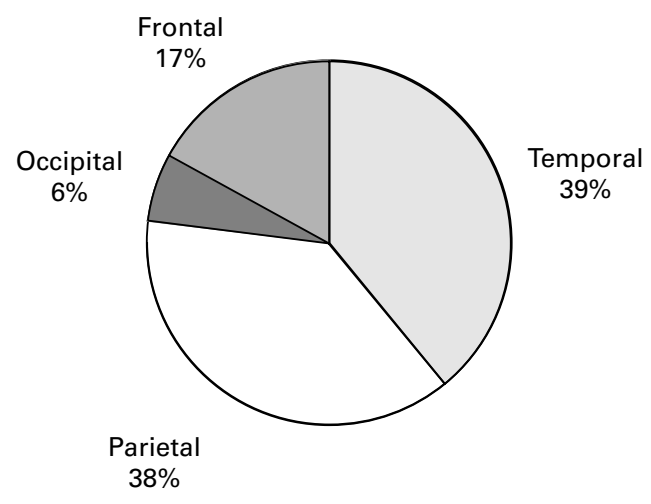

Figure 1 Location of lobar haemorrhage on initial CT.

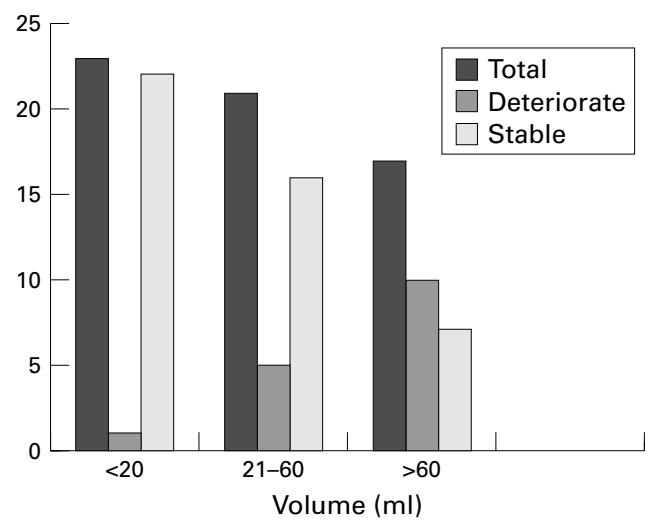

Figure 2 Volume of haemorrhage on admission CT.

\section{Results}

CLINICAL FEATURES AND NEUROIMAGING

Of the 61 patients who were eligible for analysis, $56 \%$ had a history of hypertension, and only $64 \%$ of these were treated with antihypertensive drugs. Six patients had a history of intracerebral haemorrhage and one patient had a family history of intracerebral haemorrhage. Sixty six per cent of patients presented to the emergency department within 12 hours after onset of neurological symptoms. Neurological presentation included focal neurological deficits in $42(69 \%)$, headache in $33(54 \%)$, reduced level of consciousness in 21(34\%), nausea and vomiting in $16(26 \%)$, and seizure activity in four $(6 \%)$ patients. On initial examination, $24(39 \%)$ patients presented with a GCS $\geqslant 14$. Nearly half of the patients had a systolic blood pressure $>180 \mathrm{~mm} \mathrm{Hg}$. Thirty five $(57 \%)$ presented with a motor deficit and $23(38 \%)$ had a gaze preference. Initial CT location and haemorrhage volume are graphically represented in figs 1 and 2 respectively. There was no significant difference in location between patients who deteriorated and those who remained stable. The average volume of haemorrhage was $46.7 \mathrm{ml}$ (range: $1.5-351 \mathrm{ml}$ ). Intraventricular extension was present in 15 patients $(24 \%)$.

NEUROLOGICAL DETERIORATION

Neurological deterioration occurred in 16 of $61(26 \%)$ patients. Seventy five per cent $(12 / 16)$ of patients who deteriorated presented within 6 hours of initial neurological symptoms. No significant difference in 

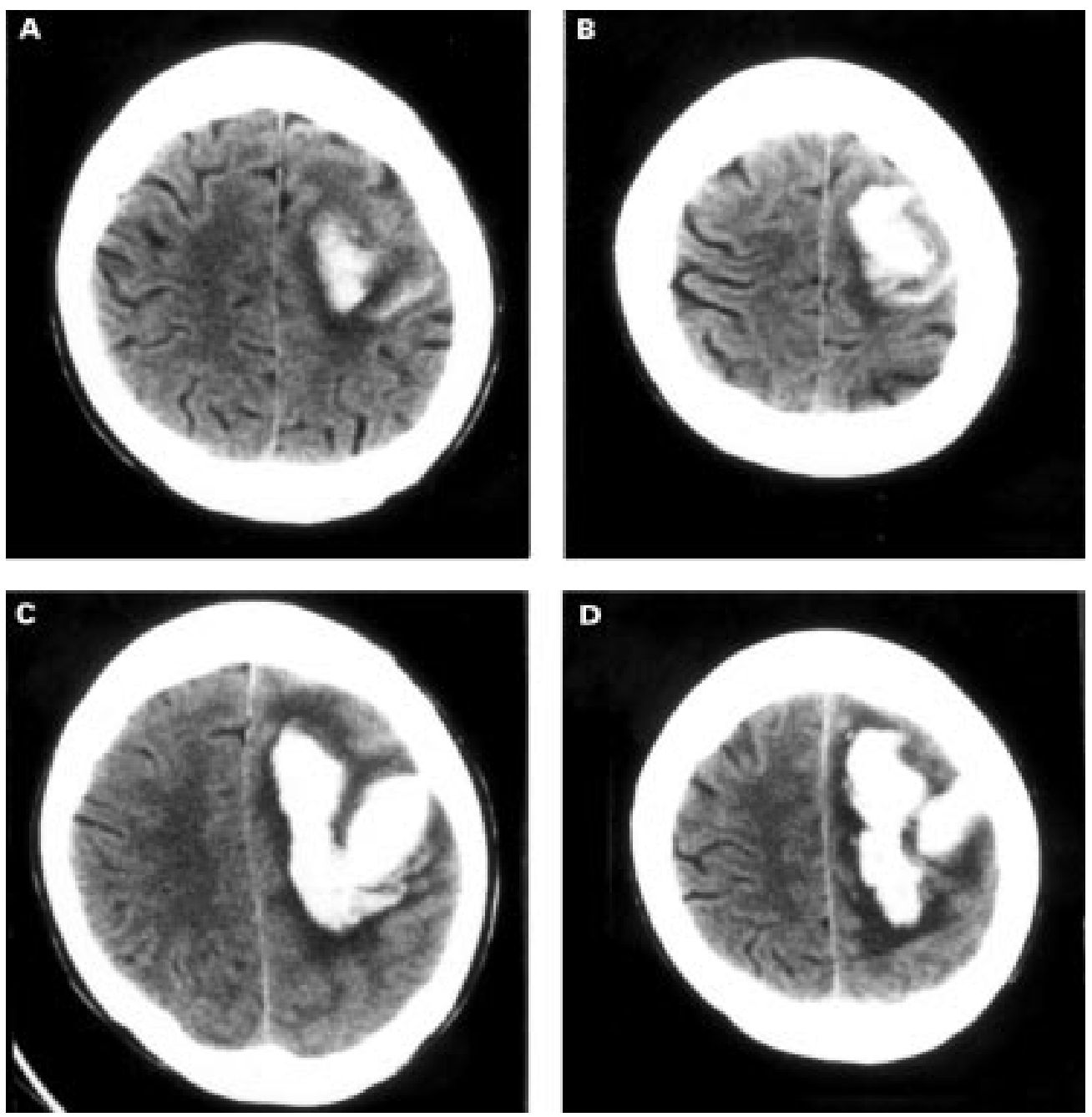

Figure 3 (A), (B) Patient admitted 1 hour after the onset of neurological symptoms with CT evidence of a left parietal haemorrhage. (C), (D) Patient subsequently deteriorated and a repeat CT performed 5 hours later showed increased volume.

demographics, medical history, clinical features at onset, or laboratory values were found between these two groups. However, $75 \%$ (12/

16) of patients who deteriorated and $24 \%$ (11/ 45) of those with stable deficits presented with a reduced level of consciousness $(\mathrm{GCS}<14$ ) $(\mathrm{p}<0.0001)$. The remainder of the initial neurological examination findings including blood pressure, gaze preference, language dysfunction, and motor weakness failed to reach significance between these two groups.

On initial CT, patients who deteriorated had a larger volume of haemorrhage (mean $91.7 \mathrm{ml}$ $v 30 \mathrm{ml})$. Sixty three per cent of deteriorators and $16 \%$ of non-deteriorators had initial haemorrhage volumes $>60 \mathrm{ml} \quad(p=0.0001)$. Mass effect as demonstrated by midline shift was more often shown by septum pellucidum midline shift than shift of the pineal gland. Patients who deteriorated showed septum pellucidum shift more often that those who did not deteriorate $(75 \%$ v 35.6\%; $\mathrm{p}=0.00089)$. Effacement of the contralateral perimesencephalic cistern $(33 \%$ v $0 \% ; \mathrm{p}=0.0005)$, indicative of horizontal displacement of the brainstem, and contralateral widening of the temporal horn $(50 \% v 0 \% ; \mathrm{p}=0.0001)$ were features present only in patients who deterio- rated. Intraventricular extension and location of the haemorrhage did not reach significance between the two groups.

Onset of neurological deterioration occurred within the first 24 hours after ictus in $75 \%$ of patients. Clinical deterioration was heralded by decreased level of consciousness in all patients (reduced GCS $>2$ points) and five patients developed new neurological deficits. Seven patients had CT at the time of deterioration. In three patients with follow up scans, deterioration occurred 3-6 hours after ictus. Two of these patients showed evidence of increased volume of haemorrhage on follow up CT (fig 3). The third showed no difference in volume or mass effect. Follow up CT was available in four patients who deteriorated 25-80 hours after onset of neurological symptoms. Repeat scans showed increased mass effect by increased septum pellucidum or pineal shift, contralateral widening of the ventricles, or evidence of herniation (fig 4).

\section{OUTCOME}

There was no statistical difference in initial therapy between those with stable deficits and those who deteriorated. Four patients with stable deficits underwent removal of 

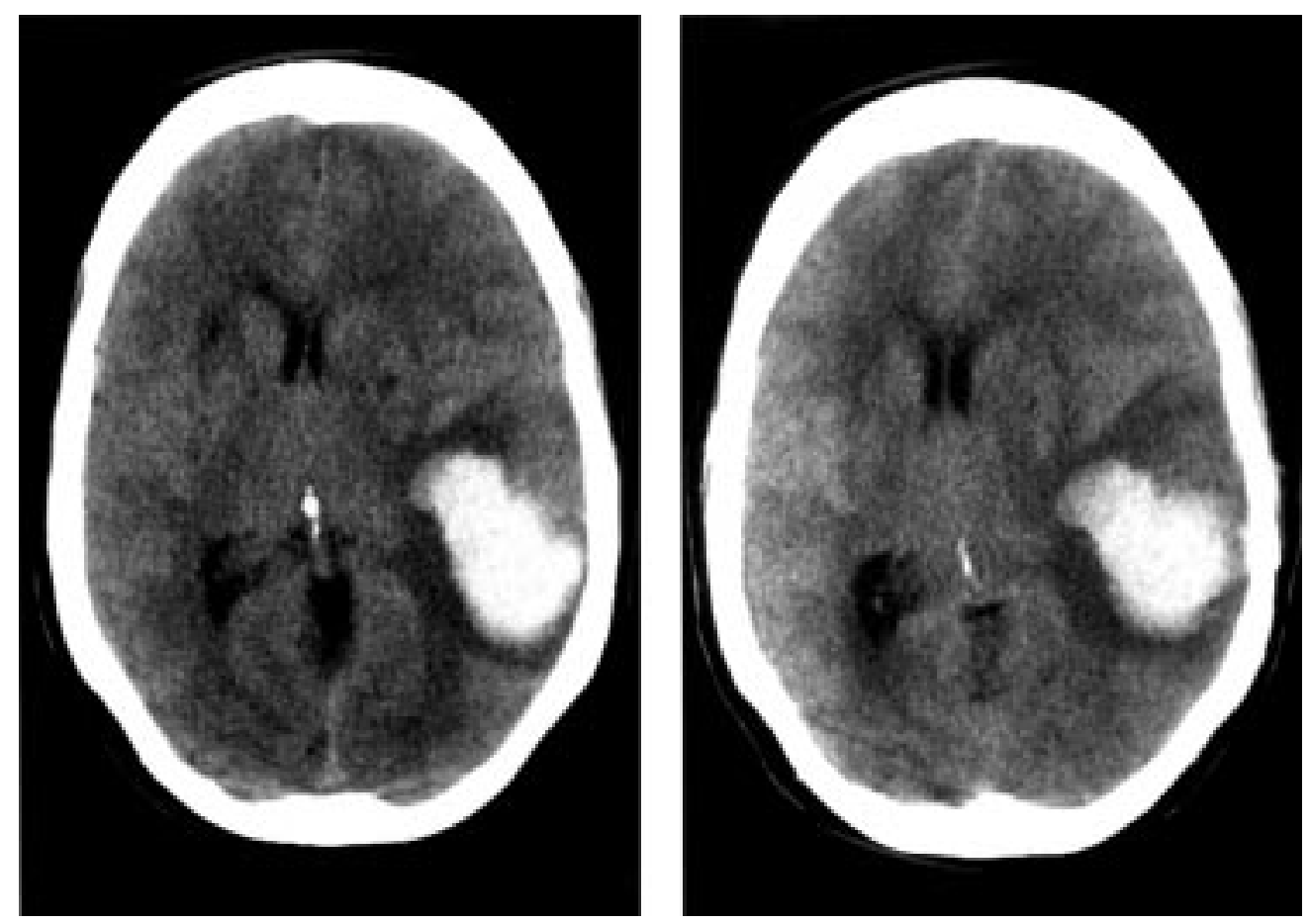

Figure 4 Clinical deterioration occurred 54 hours after ictus in this patient with a temporal lobe haemorrhage. (A) Initial CT on admission. (B) CT after deterioration. Increased mass effect is demonstrated by effacement of the supracerebellar cistern, increased shift of the pineal gland and septum pellucidum, and enlargement of the contralateral occipital horn. The haemorrhage volume remained stable.

angiographically defined arteriovenous malformations. Nineteen patients underwent angiography (two deteriorators; 17 nondeteriorators). No other patient had a pathological or angiographic diagnosis. Three patients who deteriorated underwent decompressive surgery, but remained severely disabled. The hospital course was complicated by pulmonary infection or urinary tract infection in $50 \%(8 / 16)$ of those who deteriorated and $31 \%(14 / 45)$ in those with stable deficits. Cardiovascular complications were seen in six patients (one deteriorator; five stable patients).

Patients who deteriorated were more likely to have a poor outcome or to die. Of those patients who deteriorated, $8 / 16(50 \%)$ died and $8 / 16(50 \%)$ had severe disability or persistent vegetative state. In those eight patients who deteriorated and died the mean time of death after deterioration was 3.7 days. One patient with severe disability at discharge died from a second lobar haemorrhage 7 months later. Of those patients who remained neurologically stable after presentation, 21/45 (47\%) had mild or no disability, $21 / 45$ (47\%) had moderate or severe disability, and $3 / 45(6 \%)$ died or remained in a persistent vegetative state.

\section{Discussion}

Neurological deterioration is most likely to occur within the first 48 hours after ictus. ${ }^{1-2} \quad 11-13$ It may be recognised clinically by worsening deficit, decreased level of consciousness, new deficit, or tell-tale signs of herniation. Causes include increased haemorrhage size, increasing perihaematomal oedema, development of hydrocephalus, or less likely, systemic factors.
Triage decisions in management of patients with lobar haemorrhages are often made early after presentation in the emergency department. A major clinical issue is whether deterioration, which may necessitate surgical evacuation, can be predicted with any degree of certainty.

We analysed a well defined population of lobar haemorrhages. Twenty six per cent of patients deteriorated, which is consistent with studies of deterioration in other types of intracerebral haemorrhage. ${ }^{1{ }^{14}}$ Of the clinical factors analysed, only reduced GCS on admission was associated with a significantly higher risk of deterioration. In fact, $75 \%$ of those who deteriorated had a GCS $<14$ on initial presentation compared with only $24 \%$ of those with no change in the hospital course. No other clinical features, including comorbidity, blood pressure, or laboratory studies, were predictive of deterioration. Large volume haemorrhages predicted deterioration and poor outcome. Sixty three per cent of those who deteriorated had haemorrhage volumes $>60 \mathrm{ml}$ on initial presentation. Other predictive features on initial CT included septum pellucidum shift, contralateral effacement of the perimesencephalic cistern, and contralateral widening of the temporal horn. These CT characteristics likely reflect the size, location, and space occupying effects of the haemorrhage. Close observation is warranted with consideration of early intervention when these findings are present on admission.

Risk factors for neurological deterioration have been assessed in other studies of spontaneous intracerebral haemorrhages. Estimating deterioration depends on the interval from 
ictus to initial evaluation, the definition of clinical deterioration, the population studied (comatose $v$ non-comatose), and location of the haemorrhage (supratentorial $v$ infratentorial). Mayer et al assessed the frequency, time course, and predictors of neurological deterioration in 46 non-comatose patients with supratentorial intracerebral haemorrhage, but focusing on ganglionic haemorrhages. ${ }^{1}$ It was concluded that a critical haemorrhage volume of $50 \mathrm{ml}$ on the initial CT was the best predictor of neurological deterioration. Of those patients with haemorrhage volumes greater than $50 \mathrm{ml}, 78 \%$ deteriorated.

Intracerebral haemorrhage was once considered a monophasic event. Recent studies have documented haemorrhage enlargement occurring soon after the ictus or after several days or weeks. ${ }^{515-16}$ Fujii et al studied predictors of enlargement of intracerebral haemorrhages of all types. ${ }^{17}$ His group found that presentation less than 6 hours after ictus, volume, irregular shape, and severity of liver disease predicted enlargement of intracerebral haemorrhage. In the present study, extension of the haemorrhage occurred in two patients. These patients typically presented within 1 hour of ictus and deteriorated in less than 12 hours. Neither of these patients had initial clinical or CT predictors for deterioration. It is difficult to generalise from this finding, but it is consistent with prior studies. Therefore, patients presenting very early after ictus regardless of the initial size of haemorrhage need close monitoring for potential deterioration.

Cerebral oedema may also lead to neurological deterioration. Experimental evidence has disclosed evidence of both cytotoxic and vasogenic oedema. In the present study, four patients deteriorated with CT documented evidence of increased mass effect but with no measurable change in volume. Increase in perilesional oedema was variable and difficult to measure. Often indirect features such as effacement of midline structures and obstructive hydrocephalus suggested increased mass effect. Four patients with follow up CT showing increased mass effect deteriorated 25 to 80 hours after the onset of neurological symptoms. This finding is consistent with experimental models of oedema formation in intracerebral haemorrhage which show maximal oedema development 1 to 3 days after the initial haemorrhage with resolution beginning on day $5 .{ }^{18}$ In the group of patients of Mayer et al, it was speculated that six of nine lobar haemorrhages deteriorated from oedema. The average ictus to initial evaluation in that study was 13.5 hours but it is possible that the potential contribution of haemorrhage enlargement had been underestimated. ${ }^{19}$

None of our patients deteriorated from acute hydrocephalus. The location of the haemorrhage would account for that because only after appreciable enlargement does the ventricular system become compromised.

The analysis of outcome in our patients is comparable with other reported studies. $^{1-2}$ Patients who deteriorate have increased mor- bidity and mortality. Although this seems intuitive, deterioration is often used as a surgical indication. Three patients underwent surgical evacuation and decompression after deterioration. At discharge all three were severely disabled. One of the three presented alert with a large volume haemorrhage. It remains to be seen whether early surgical intervention in selected patients could improve morbidity or mortality. ${ }^{20-24}$

Certain limitations of our retrospective study must be emphasised. Our study population was truncated by eliminating non-comatose patients (who have already deteriorated), those presenting after 48 hours (period of maximum oedema), and those patients with an underlying symptomatic cause of haemorrhage (trauma, tumour, coagulopathy, ruptured aneurysm). In addition, less than half of our patients who deteriorated had follow up CT at the time of deterioration, which limits the determination of mechanism by CT. Due to the limited number of patients, multivariate analysis was not performed.

Twenty six per cent of patients presenting with acute lobar haemorrhage deteriorated and these patients had increased morbidity and mortality. Patients presenting early need close observation within the first 24 hours as deterioration from enlarging haemorrhage may develop. Haemorrhage enlargement may not be predicted from initial CT findings. Patients presenting with reduced level of consciousness and large volume haemorrhage by CT are likely to deteriorate from oedema. Controversy still exists in the medical management of this type of haemorrhage and surgical results have been variable. ${ }^{80-25}$ Further studies are needed to elucidate the pathophysiology of lobar intracerebral haemorrhages and the usefulness of early, surgical intervention before deterioration.

1 Mayer S, Sacco RL, Shi T, et al. Neurologic deterioration in noncomatose patients with supratentorial intracerebral hemorrhage. Neurology 1994;44:1379-84.

2 Qureshi AI, Safdar K, Weil EJ, et al. Predictors of early deterioration and mortality in black Americans with spontaneous intracerebral hemorrhage. Stroke 1995;26: taneous

3 Broderick JP. Clinical and radiographic dynamics of ICH. Presented at the 20th International foint Conference on Stroke and Cerebral Circulation. Charleston, SC: February, 1995.

4 Broderick JP, Brott T, Kothari, et al. Very early edema growth not significantly associated with neurologic deterioration in patients with intracerebral hemorrhage [abstract 145]. Stroke 1995;26:184.

5 Broderick JP, Brott TG, Tomsick T, et al. Ultra-early evaluation of intracerebral hemorrhage. $\mathcal{F}$ Neurosurg 1990;72: 195-9.

6 Bae HG, Lee KS, Yun IG, et al. Rapid expansion of hypertensive intracerebral hemorrhage. Neurosurgery 1992;31: 35-41.

7 Chen ST, Chen SD, Hsu CY, et al. Progression of hypertensive intracerebral hemorrhage. Neurology 1989;39:150914.

8 Kaneko M, Tanaka K, Shimada T, et al. Long-term evaluation of ultra-early operation for hypertensive intracerebral hemorrhage in 100 cases. F Neurosurg 1983;58: 838-42.

9 Kothari RU, Brott T, Broderick JP, et al. The ABC's of measuring intracerebral hemorrhage and volumes. Stroke 1996;27:1304-5.

10 Jennett B, Snoek J, Bond MR, et al. Disability after severe head injury: observations on use of the Glasgow outcome head injury: observations on use of the Glasgow outcor
scale. F Neurol Neurosurg Psychiatry 1981;44:285-93.

11 Broderick JP, Brott TG, Duldner JE, et al. Volume of intracerebral hemorrhage: a powerful and easy-to-use predictor of 30 day mortality. Stroke 1993;24:987-93. 
12 Broderick J, Brott T, Tomsick T, et al. Management of intracerebral hemorrhage in a large metropolitan populaintracerebral hemorrhage in a large
tion. Neurosurgery 1994;34:882-7.

13 Wijdicks EFM. Supratentorial intraparenchymal hemorrhage. In: Wijdicks EJM. The clinical practice of critical care neurology. Philadelphia: Lippincott-Raven, 1997 158-72.

14 Jorgensen HS, Nakayama H, Raaschou HO, et al. Effect of blood pressure and diabetes on stroke in progression. Lancet 1994;344:156

15 Fehr MA, Anderson DC. Incidence of progression or rebleeding in hypertensive intracerebral hemorrhage. $\mathcal{F}$ Stroke Cerebrovasc Dis 1991;1:111-16.

16 Wijdicks EFM, Fulgham, JR. Acute fatal deterioration in putaminal hemorrhage. Stroke 1995;26:1953-5.

17 Fujii Y, Tanaka R, Takeuchi S, et al. Hematoma enlargement in spontaneous intracerebral hemorrhage. $f$ Neurosurg 1994;80:51-7.

18 Lee KR, Betz AL, Kim S, Keep RF, Hoff JT. The role of the coagulation cascade in grain edema formation after intracerebral hemorrhage. Acta Neurochir 1996;138:396-40.

19 Fulgham JR, Wijdicks EFM. Deterioration with hemorrhage. Neurology 1995;45:602.
20 Juvela S, Heiskanen O, Poranen A, et al. The treatment of spontaneous intracerebral hemorrhage: a prospective randomized trial of surgical
rosurg 1989;70:755-8.

21 Auer LM, Deinsberger W, Niederkorn K, et al. Endoscopic surgery versus medical treatment for spontaneous intracerebral hematoma: a randomized study. $\mathcal{F}$ Neurosurg 1989;70: $530-5$.

22 Unwin DH, Batjer HH, Greenlee RG. Management controversy: medical versus surgical therapy for spontaneous intracerebral hemorrhage. Neurosurg Clin N Am 1992; 3:533-7.

23 McKissock W, Richardson A, Taylor J. Primary intracerebral hemorrhage. A controlled trial of surgical and conservative treatment in 180 unselected cases. Lancet 1961;ii:221-6.

24 Minakawa T, Takeuchi SH, Sasaki O, et al. Surgical experience with massive lobar haemorrhage caused by cerebral amyloid angiopathy. Acta Neurochir (Wien) 1995;132:4852 .

25 Masdeu JC, Rubino FA. Management of lobar intracerebral hemorrhage: medical or surgical. Neurology 1984;34:381-3. 